\title{
Abdominal Viscera Migration Performing Hemodynamic Instability after Esophagectomy: A Case Report
}

\author{
Ian Torres de Lima ${ }^{a}$ Edno Tales Bianchi ${ }^{b}$ Gabriel Lunardi Aranha ${ }^{a}$ \\ Beatriz Camargo Azevedo $^{\mathrm{b}}$ Guilherme Naccache Namur ${ }^{\mathrm{b}}$ \\ Jaime Arthur Pirola Kruger ${ }^{b}$ \\ aDepartment of General Surgery, Hospital Israelita Albert Einstein, São Paulo, Brazil; ${ }^{\text {b}}$ Department of Gastrointestinal \\ Oncology Surgery, Hospital Israelita Albert Einstein, São Paulo, Brazil
}

\section{Keywords}

Esophageal cancer · Esophagectomy · Hiatal hernia

\section{Abstract}

Hiatal hernia is a rare postoperative complication of esophagectomy in the treatment of esophageal cancer. Although rare, its incidence increased after the establishment of minimally invasive surgical techniques. The patient is usually oligosymptomatic, and the diagnosis is made in the late postoperative period, during outpatient follow-up. The initial presentation of hiatus hernia with hemodynamic instability is a rare condition that has never been described in the literature before. In the following report, we address the clinical picture, diagnosis, and treatment for this condition, discussing the main nuances of the literature.

(C) 2021 The Author(s)

Published by S. Karger AG, Basel

karger@karger.com www.karger.com/gat

Karger"

BOPEN ACCESS
C) 2021 The Author(s).

Published by S. Karger AG, Basel

This is an Open Access article licensed under the Creative Commons Attribution-NonCommercial-4.0 International License (CC BY-NC) (http://www.karger.com/Services/OpenAccessLicense), applicable to the online version of the article only. Usage and distribution for commercial purposes requires written permission.

\section{Introduction}

The minimally invasive surgical treatment for esophageal cancer has gained ground in recent years, as it involves fewer complications in the postoperative period, when compared to the open approach. However, it is related to a higher incidence of hiatal hernia $(\mathrm{HH})$, a rare complication after esophagectomy [1]. Although rare, $\mathrm{HH}$ needs repair in an emergency regimen between 22 and $54 \%$ of cases - depending on the study $[2,3]$. In addition, urgent repair has a significant morbidity rate $25 \%$, reaching $38.6 \%$ [2].

The most accepted initial approach to this complication appears to be videolaparoscopy [2-4]. We report a case in which increased abdominal pressure may have contributed to the acute migration of abdominal contents through the esophageal hiatus after minimally invasive esophagectomy, leading to an atypical clinical picture with hemodynamic manifestations. The clinical management and surgical correction adopted will also be covered.

Correspondence to:

Ian Torres de Lima, iantorres@outlook.com.br 


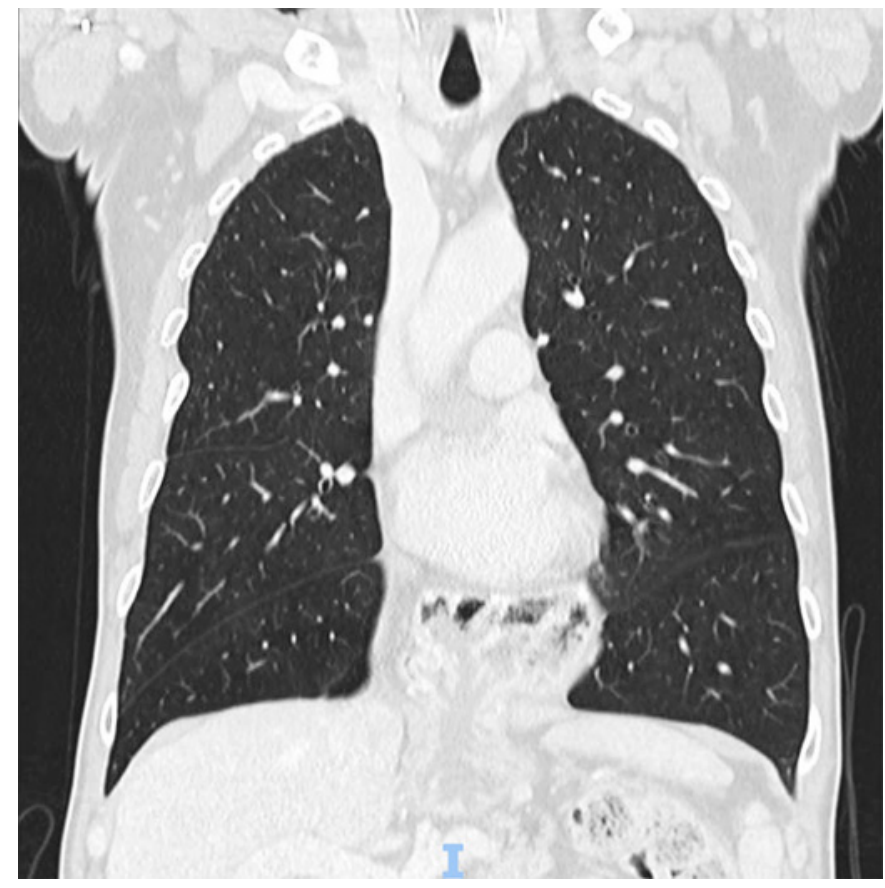

Fig. 1. Preoperative chest tomography (coronal section). Evidence of massive hiatal gastric hernia.

\section{Case Report}

The patient is a 61-year-old male, who is alcoholic (consumption of distillates daily since the age of 18) with no smoking history. His father died at 67 years of age from stomach cancer, and his mother died at 84 years of age from esophageal cancer. Other relevant background includes chronic constipation with eventual need for enteroclysis and hip prosthesis due to traumatic fracture - which limits ambulation.

Due to epigastric pain and weight loss, an upper digestive endoscopy was performed (on November 11, 2019), which showed columnar-looking epithelium at $30 \mathrm{~cm}$ from the upper dental arch, extending to the esophagogastric transition. In the esophagogastric transition, there was an elevated and hardened lesion affecting $30 \%$ of the organ lumen. Biopsies pointed Barrett's esophagus with low-grade dysplasia and ulcerated adenocarcinoma (histological grade 2 ).

CT scans (chest, abdomen, head, and neck) for staging (held on March 14, 2019) concluded a T2N0M0 tumor (shown in Fig. 1, 2). Additional finding included bulky hiatal gastric hernia, containing part of the body and fundus of the stomach. Preoperative echocardiogram (held on April 5, 2019) showed an ejection fraction of $64 \%$, with normal cardiac morphology and dynamics (PSAP 22 $\mathrm{mm} \mathrm{Hg}$ ).

A video-assisted esophagectomy was performed with thoracoscopy and laparoscopy, using a gastric tube for esophageal reconstruction. Intraoperatively, the chest is usually drained bilaterally, and a nasoenteral tube is passed, which remains until the 7 th postoperative day.

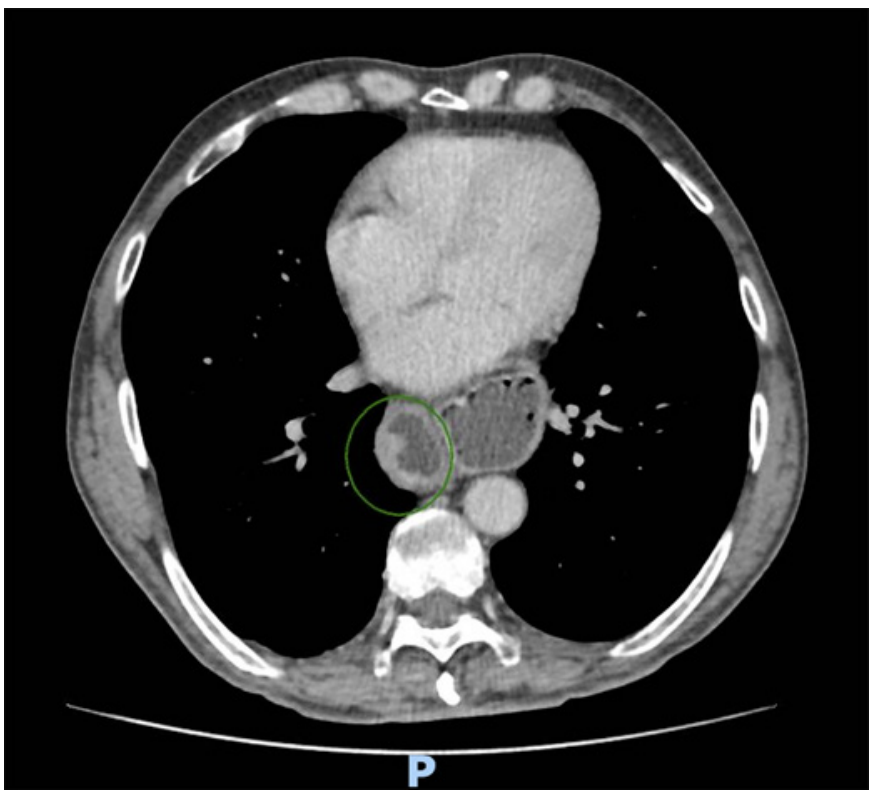

Fig. 2. Preoperative chest tomography (cross section). Evidence of massive hiatal gastric hernia and irregular and asymmetrical parietal thickening of the distal esophagus.

The patient was in the ICU for the first 3 days after surgery. Figure 3 shows a chest X-ray after the procedure. In the 4 th postoperative period, after performing a Valsalva maneuver to try to evacuate, the patient developed sudden chest pain, associated with tachycardia, hypotension, jugular turgescence, and decreased chest auscultation - at the right hemithorax. A new radiograph was performed (shown in Fig. 4), showing intestinal migration in the right hemithorax with mediastinal deviation.

Urgent laparotomy was indicated. During surgery, the transverse colon and the small intestine were found herniated. After content reduction, the patient evolved with complete resolution of tachycardia and hypotension. A 2-point suture hiatoplasty, with nonabsorbable suture (prolene), was performed.

The postoperative recovery took place in the ICU, involving a quick improvement (shown in Fig. 5). There was no change in myocardial necrosis markers or any other cardiovascular complications. The patient was discharged to the infirmary after 2 days on an enteral diet. Clinical measures for constipation were adopted, aiming to reduce intra-abdominal pressure during evacuation. Oral diet was offered on the 7 th postoperative day. On the 10th postoperative day, the patient was discharged home with exams showing absence of hernia and with good acceptance of the creamy oral diet as well as regular intestinal transit. A new control chest tomography was performed after 1 year, with no evidence of herniation of the abdominal contents to the chest (shown in Fig. 6, 7). 


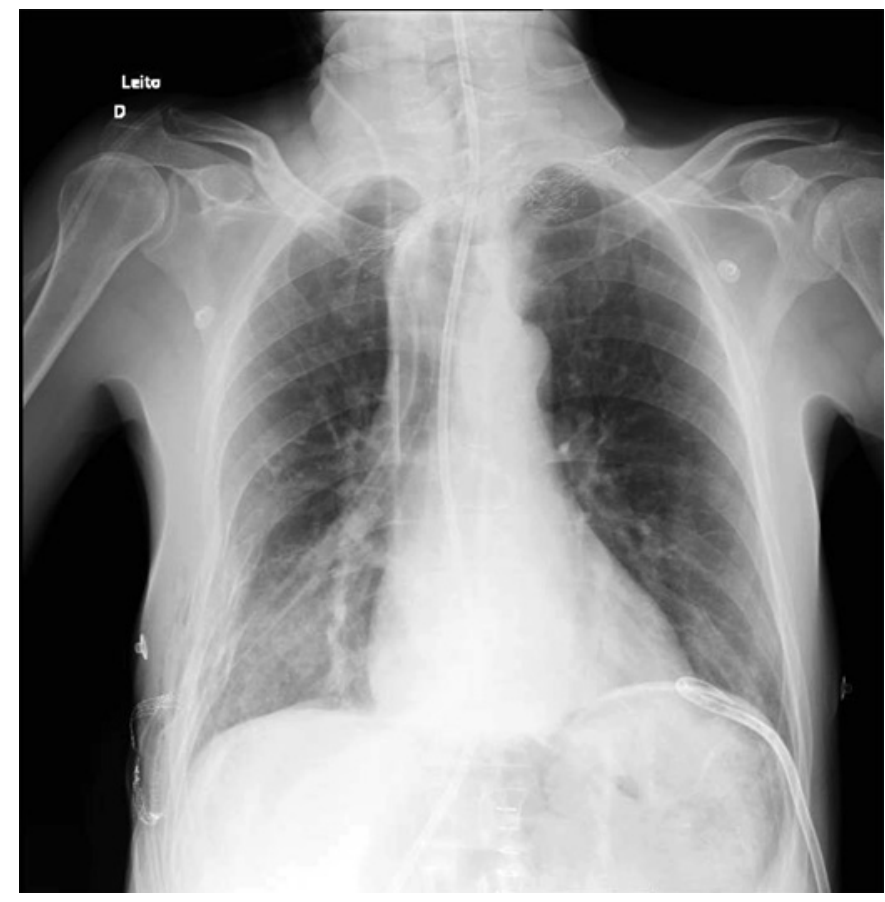

Fig. 3. Chest X-ray in bed (1st postoperative period of esophagectomy). Free costophrenic sinuses and opacities in the right hemithorax, compatible with the postoperative period. Bilateral chest drainage and nasoenteral tube in usual position.

\section{Discussion}

$\mathrm{HH}$ is a rare complication after surgical treatment of esophageal cancer. Its incidence varies between open surgical techniques (1\%) and minimally invasive by videolaparoscopy $(4.5 \%)$ - reaching up to $19 \%$ in robotic surgery [2]. The minimally invasive approach promotes less formation of adhesions; moreover, it involves application of intracavitary gas and wider dissection of the esophageal hiatus, and such characteristics may be related to the higher incidence. The Ivor-Lewis technique, among the minimally invasive techniques, has been shown to be related to a higher incidence of $\mathrm{HH}$; however, there is an insufficient number of studies to attest the real incidence among the different techniques $[1,2,5]$.

Although rare, its occurrence has a growing trend due to the increased survival in esophageal cancer and the greater use of minimally invasive techniques [5]. The average time between esophagectomy and the time of diagnosis of symptomatic $\mathrm{HH}$ is approximately 8 months - it can vary between 1 and 32 months [2,3]. The most common symptom presented in early diagnosis (within 30 days from esophagectomy) is respiratory distress, and the

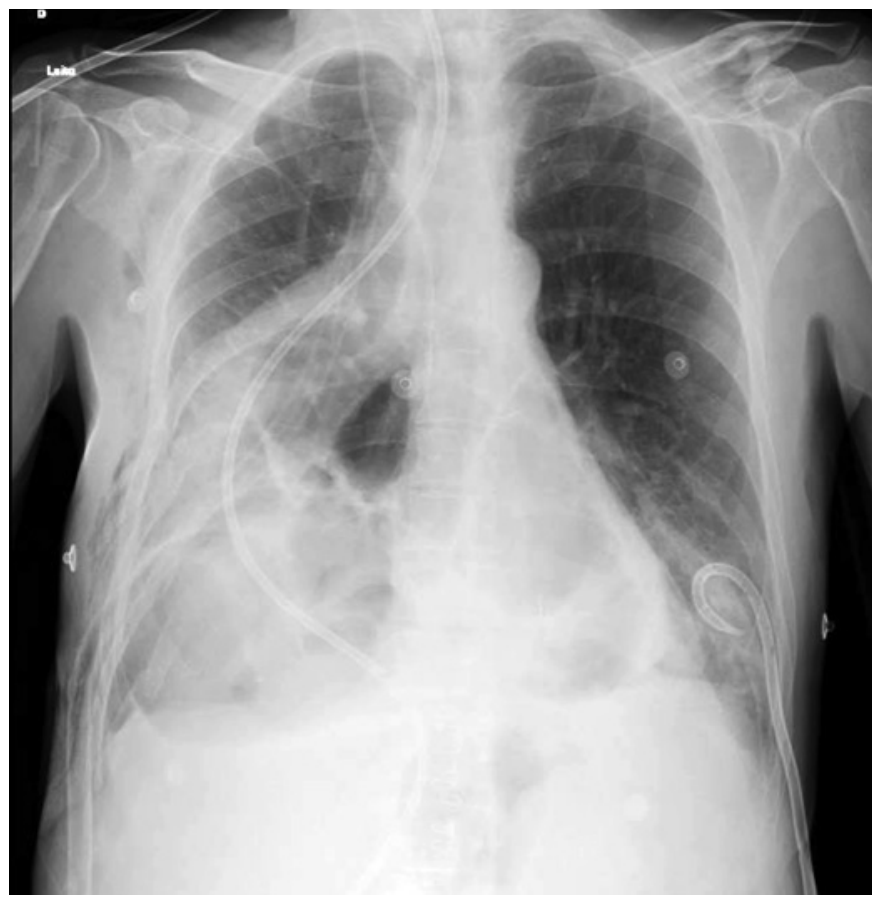

Fig. 4. Chest X-ray in bed (4th postoperative day). Great distortion of the gastric tube architecture, with the presence of abdominal contents in the chest and atelectasis of the right lower lobe of the lung.

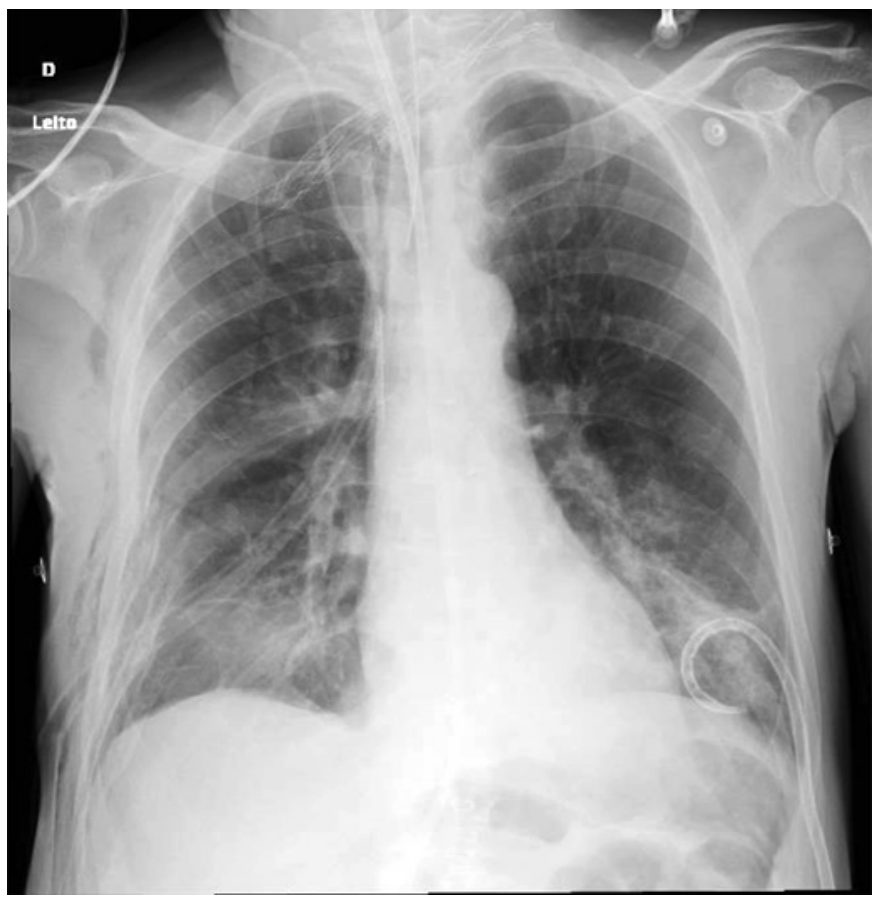

Fig. 5. Chest X-ray in bed (1st postoperative day of surgical re-approach). Preserved gastric tube architecture and absence of abdominal contents in the chest. Bilateral chest drain and nasoenteral tube in usual position. 


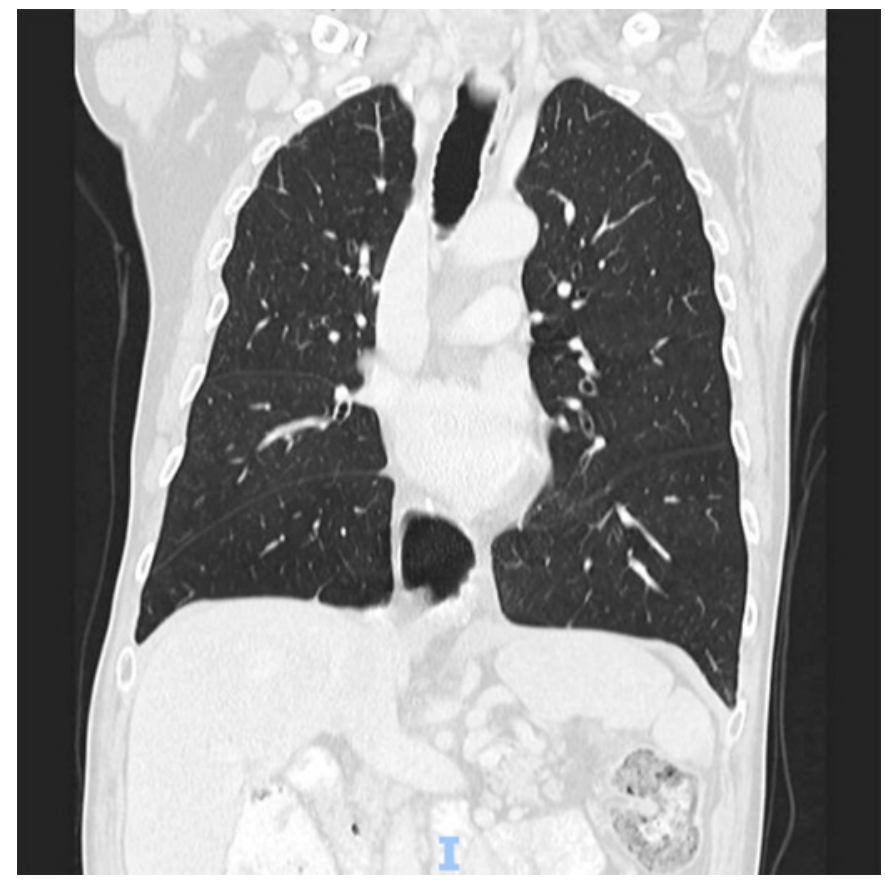

Fig. 6. Chest tomography, coronal section, 8 months after the surgical procedure (esophagectomy). No evidence of hiatal hernia.

most common symptoms in late diagnosis are thoracoabdominal pain and dysphagia [2]. Hemodynamic instability is an atypical symptom that implies greater severity and may be related to bowel ischemia and perforation [1]. In the present report, after herniation, the patient evolved with restrictive shock by cardiac compression, mimicking a cardiogenic shock, which has never been described in the literature before.

Computed tomography is widely used for $\mathrm{HH}$ diagnosis $[2,3]$. However, in symptomatic patients, the X-ray alone is sufficient to indicate surgical correction. The performance of imaging exams in the postoperative period does not occur routinely, and they are mainly requested in symptomatic patients, when there is a clinical suspicion of complications. Thus, many cases are underdiagnosed, not reflecting the real incidence of this condition $[1,2]$.

Some authors advocate the execution of imaging exams as a routine in the postoperative period of esophagectomy $[2,4,5]$. This makes diagnosis possible in asymptomatic patients, as they may become symptomatic over time, increasing the chance of complications. Thus, some authors advocate elective correction regardless of the presence of symptoms $[2,5]$. Such approach is not widely accepted, since there is a high morbidity involving

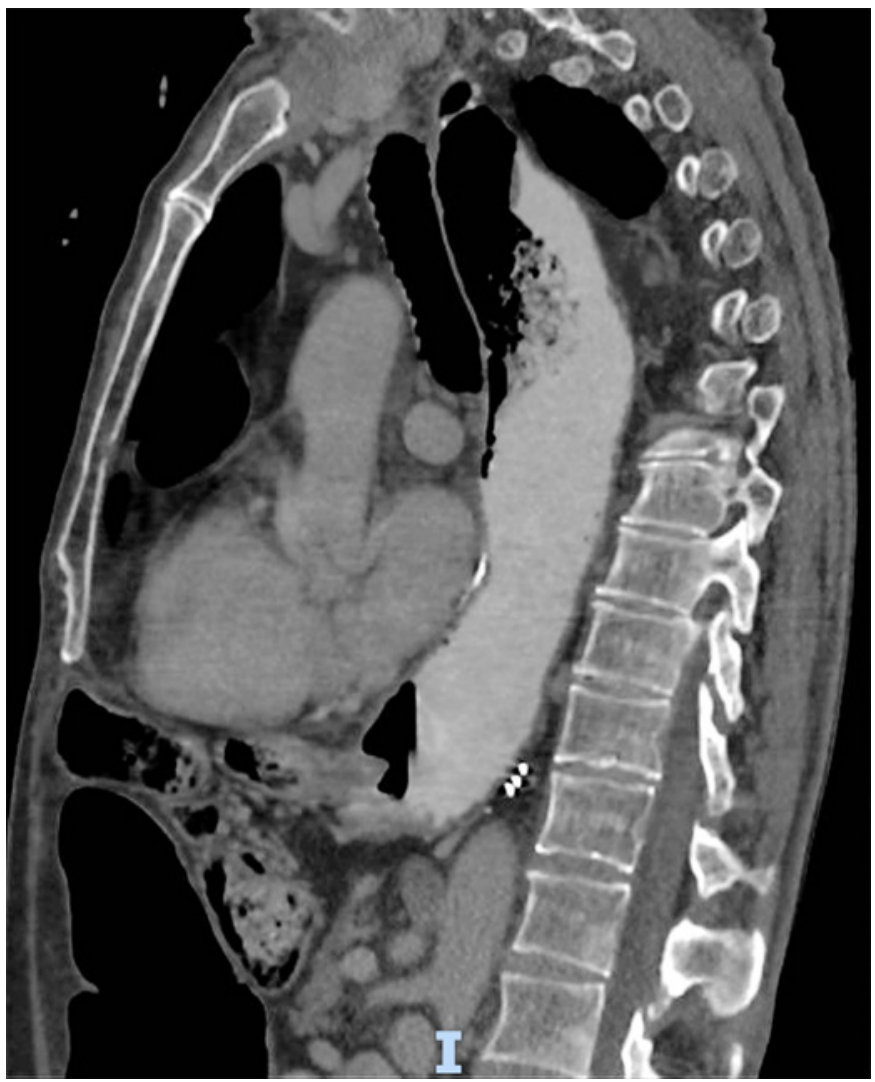

Fig. 7. Chest tomography, sagittal section, 8 months after the surgical procedure (esophagectomy). No evidence of hiatal hernia. Stasis content and no other findings.

the repair. Therefore, the indication for treatment preponderates when there are symptoms, especially in the presence of cardiorespiratory instability. The conservative approach seems safe in asymptomatic patients $[2,4$, 5].

Regarding $\mathrm{HH}$ rectification, the use of mesh is not unanimous, and some studies have obtained a lower recurrence rate with the use of routine mesh, when compared to isolated suture with nonabsorbable thread (12.1\% and 20.5\%, respectively) [1]. However, several other studies have not identified a significant difference [2]. However, when there is a wide defect, where a tension-free technique cannot be performed by isolated primary closure, the mesh use is legitimized $[2,3]$. Circular screen layout should be avoided, preventing direct contact with the neoesophagus and its vascular supply [2].

The repair of $\mathrm{HH}$ after esophagectomy involves high morbidity, varying between 11 and 38.6\%. Therefore, the greatest effort should be on preventing this complication. Preventive measures have already been described, such as 
cruroplasty with the use of a mesh in the initial approach of esophagectomy. Some centers applied the use of a prophylactic mesh for all patients; however, they did not observe a significant drop in the global incidence of $\mathrm{HH}$ [2].

One aspect rarely addressed in these cases is intra-abdominal pressure as a cause of migration. It is already known that the increase in the thoracoabdominal pressure gradient can cause gastroesophageal reflux, especially in patients with a high BMI as a cause of increased abdominal pressure or in patients with certain lung diseases $[6,7]$. It can be inferred that the same mechanism could increase the risk of esophageal migration after esophagectomy. In the present case, the increase in abdominal pressure secondary to constipation led to the migration of abdominal contents. Therefore, this risk could have been reduced by installing laxative measures before surgery.

In abdominal hernias, the use of botulinum toxin in the abdominal wall has been described preoperatively to decrease intra-abdominal pressure [8]. This toxin blocks the release of acetylcholine in the terminal cholinergic nerve [9], causing a temporary loss of muscle tone, stretching and decreasing muscle thickness $[8,10]-\mathrm{a}$ mechanism capable of decreasing intra-abdominal pressure, avoiding herniation [10-12]. Such a strategy has already been described in the treatment of bulky hiatus hernia and could also be used as a tactic to prevent the migration of abdominal viscera to the chest after esophagectomy [13].

\section{Conclusion}

Studies involving $\mathrm{HH}$ after esophagectomy in esophageal cancer have several limitations because they are mainly retrospective. Despite involving a higher incidence rate of $\mathrm{HH}$, the minimally invasive approach to esophagectomy is superior to the open approach, as it involves shorter hospital stay, less bleeding volume, and less perioperative complications $[1,2,4]$. The repair of $\mathrm{HH}$ by videolaparoscopy seems to be more adequate; however, the routine use of the mesh is still controversial $[2,3]$.

Prevention techniques seem to be a great strategy; however, so far, they have not shown effectiveness in studies [2]. This problem highlights the need for new studies aiming to elucidate a better preventive strategy. At the present, it remains for the multidisciplinary team involved in the treatment of this group of patients to pay attention to this potentially life-threatening condition during the postoperative follow-up.

Manifestation of Hiatal Hernia Simulating

Cardiogenic Shock after Esophagectomy

\section{Statement of Ethics}

Written and informed consent has been taken from the patient in order to publish the manuscript contents, including images. Approval number from the Plataforma Brasil Ethics Committee: 66831717.2 .0000 .0030 .

\section{Conflict of Interest Statement}

The authors have no conflicts of interest to declare.

\section{Funding Sources}

The authors did not receive any funding.

\section{Author Contributions}

Ian Torres de Lima contributed substantially to the design, structuring, and drafting of the work. Edno Tales Bianchi contributed substantially in acquisition and critically analyzing the data, adding from the critical point of view the vast experience on the subject. Gabriel Lunardi Aranha contributed substantially in evaluation, structuring of images, and drafting the work. Beatriz Camargo Azevedo contributed substantially in describing the images as well as reviewing the discussion. Guilherme Naccache Namur contributed substantially in critically reviewing the available literature and structuring the conclusion. Jaime Arthur Pirola Kruger contributed substantially in revising the final version of the work and structuring in the patterns required for publication.

\section{Data Availability Statement}

All data generated or analyzed during this study are included in this article. Further enquiries can be directed to the corresponding author.

References

1 Gooszen JAH, Slaman AE, van Dieren S, Gisbertz SS, van Berge Henegouwen MI. Incidence and treatment of symptomatic diaphragmatic hernia after esophagectomy for cancer. Ann Thorac Surg. 2018 Jul;106(1): 199-206.

2 Oor JE, Wiezer MJ, Hazebroek EJ. Hiatal hernia after open versus minimally invasive esophagectomy: a systematic review and meta-analysis. Ann Surg Oncol. 2016 Aug;23(8): 2690-8.

3 Gust L, Nafteux P, Allemann P, Tuech JJ, El Nakadi I, Collet D, et al. Hiatal hernia after oesophagectomy: a large European survey. Eur J Cardiothorac Surg. 2019 Jun;55(6): 1104-12. 
4 Marchesi F, Dalmonte G, Morini A, Annicchiarico A. Laparoscopic repair of a giant hiatal hernia after minimally invasive oesophagectomy. Ann R Coll Surg Engl. 2020 Jul; 102(6):e130-2.

5 Brenkman HJ, Parry K, Noble F, van Hillegersberg R, Sharland D, Goense L, et al. Hiatal hernia after esophagectomy for cancer. Ann Thorac Surg. 2017 Apr;103(4):1055-62.

6 de Vries DR, van Herwaarden MA, Smout AJ, Samsom M. Gastroesophageal pressure gradients in gastroesophageal reflux disease: relations with hiatal hernia, body mass index, and esophageal acid exposure. Am J Gastroenterol. 2008 Jun;103(6):1349-54.
7 Ayazi S, DeMeester SR, Hsieh CC, Zehetner J, Sharma G, Grant KS, et al. Thoraco-abdominal pressure gradients during the phases of respiration contribute to gastroesophageal reflux disease. Dig Dis Sci. 2011 Jun;56(6): 1718-22.

8 Ibarra-Hurtado TR, Nuño-Guzmán CM, Echeagaray-Herrera JE, Robles-Vélez E, de Jesús González-Jaime J. Use of botulinum toxin type a before abdominal wall hernia reconstruction. World J Surg. 2009 Dec;33(12): 2553-6.

9 Montecucco C, Schiavo G. Mechanism of action of tetanus and botulinum neurotoxins. Mol Microbiol. 1994 Jul;13(1):1-8.

10 Farooque F, Jacombs AS, Roussos E, Read JW, Dardano AN, Edye M, et al. Preoperative abdominal muscle elongation with botulinum toxin A for complex incisional ventral hernia repair. ANZ J Surg. 2016 Jan-Feb;86(1-2): 79-83.
11 Riley DS, Barber MS, Kienle GS, Aronson JK, von Schoen-Angerer $\mathrm{T}$, Tugwell $\mathrm{P}$, et al. CARE guidelines for case reports: explanation and elaboration document. J Clin Epidemiol. 2017;89:218-35.

12 Soltanizadeh S, Helgstrand F, Jorgensen LN. Botulinum toxin $\mathrm{A}$ as an adjunct to abdominal wall reconstruction for incisional hernia. Plast Reconstr Surg Glob Open. 2017 Jun; 5(6):e1358.

13 Tustumi F, Bianchi ET, Szachnowicz S, Cedro RM, de Miranda Neto AA, Morrell ALG, et al. Preoperative botulinum toxin type A: a case report of a proposed new strategy for giant hiatal hernia management. In: Bianchi ET, editor. Clin Case Rep. 2020;8(12):3412-15. 